\title{
A DUAL VERSION OF HUPPERT'S CONJECTURE ON CONJUGACY CLASS SIZES *
}

\author{
Zeinab Akhlaghi ${ }^{1}$, Maryam Khatami ${ }^{2}$, Tung Le $^{3}$, \\ Jamshid Moori ${ }^{3}$, Hung P. Tong-Viet ${ }^{4}$ \\ ${ }^{1}$ Faculty of Math. and Computer Sci., \\ Amirkabir University of Technology (Tehran Polytechnic), 15914 Tehran, Iran \\ e-mail: z_akhlaghi@aut.ac.ir \\ 2 Department of Mathematics, University of Isfahan, \\ Isfahan, 81746-73441, Iran \\ e-mail: m.khatami@ui.sci.ac.ir \\ ${ }^{3}$ School of Mathematical Sciences, North-West University (Mafikeng) \\ Mmabatho 2735, South Africa \\ e-mail: lttung96@yahoo.com \\ e-mail: Jamshid.Moori@nwu.ac.za \\ and \\ ${ }^{4}$ Fakultät für Mathematik, Bielefeld Universität \\ Postfach 1001 31, D-33501 Bielefeld, Germany \\ e-mail: ptongviet@math.uni-bielefeld.de
}

\begin{abstract}
In [1], a conjecture of J.G. Thompson for $\operatorname{PSL}_{n}(q)$ was proved. It was shown that every finite group $G$ with the property $\mathbf{Z}(G)=1$ and $\operatorname{cs}(G)=\operatorname{cs}\left(\operatorname{PSL}_{n}(q)\right)$ is isomorphic to $\operatorname{PSL}_{n}(q)$ where $\operatorname{cs}(G)$ is the set of conjugacy class sizes of $G$. In this article we improve this result for $\operatorname{PSL}_{2}(q)$. In fact we prove that if $\operatorname{cs}(G)=\operatorname{cs}\left(\operatorname{PSL}_{2}(q)\right)$, for $q>3$, then $G \cong \operatorname{PSL}_{2}(q) \times A$, where $A$ is abelian. Our proof does not depend on the classification of finite simple groups.
\end{abstract}

keywords: Conjugacy classes, Simple groups, Huppert's conjecture.

\section{Introduction}

Let $G$ be a finite group and $\mathbf{Z}(G)$ be its center. For $x \in G$, assume that $x^{G}$ is the conjugacy class of $G$ containing $x$ and $C_{G}(x)$ denotes the centralizer of $x$ in $G$. We use $\operatorname{cs}(G)$ for the set of all conjugacy class sizes of $G$, that is $\operatorname{cs}(G)=\left\{\left|g^{G}\right|: g \in G\right\}$. In this article we are concerned with the following open conjecture (Conjecture 1$)$ :

Conjecture 1. (Dual of Huppert's Conjecture). Let $G$ be a finite group and let $H$ be a nonabelian simple group. If $\operatorname{cs}(G)=\operatorname{cs}(H)$, then $G \cong H \times A$, where $A$ is abelian.

*Mathematics Subject Classification: 20E45, 20 D05. 
This is exactly the dual of Huppert's Conjecture on character degree sets of nonabelian simple groups, which was formulated by B. Huppert in the late 1990. If $\operatorname{cd}(G)$ denotes the character degree set of $G$, then we have:

Conjecture 2. (Huppert's Conjecture). Let $G$ be a finite group and let $H$ be a nonabelian simple group. If $\operatorname{cd}(G)=\operatorname{cd}(H)$, then $G \cong H \times A$, where $A$ is abelian.

Note that Dual of Huppert's conjecture is an extension of Thompson's conjecture:

Conjecture 3. (Thompson's Conjecture). Let $G$ be a finite group with trivial center and let $H$ be a nonabelian simple group. If $\operatorname{cs}(G)=\operatorname{cs}(H)$, then $G \cong H$.

In [1], using the classification of finite simple groups, it is shown that $\operatorname{PSL}_{n}(q)$ satisfies Thompson's conjecture. In this article we want to improve this result and we will show that $\operatorname{PSL}_{2}(q)$ satisfies Dual of Huppert's Conjecture. At first we obtained the result by using the main theorem in [1]. However, we have chosen to avoid direct reference to the main theorem of [1], in order to keep our proofs independent on the classification of the finite simple groups. The proof is divided into two parts. First, we consider a finite group $G$ such that $\operatorname{cs}(G)=\operatorname{cs}\left(\operatorname{PSL}_{2}(q)\right)$, when $q$ is even and to get the result in this case, we will use the classification of $F$-groups. By an $F$-group we mean a family of all finite groups $G$ in which for any $x, y \in G-\mathbf{Z}(G)$, if $C_{G}(x) \leq C_{G}(y)$, then $C_{G}(x)=C_{G}(y)$. For a noncentral element $x \in G$, the centralizer $C_{G}(x)$ is said to be free if it is both maximal and minimal among all the proper centralizers of $G$. A group $G$ is $F$-group, if and only if all of the centralizers of its non-central elements are free. The $F$-groups have been classified by Rebmann [11]. Secondly we study a group $G$ such that $\operatorname{cs}(G)=\operatorname{cs}\left(\mathrm{PSL}_{2}(q)\right)$, for odd number $q$. In this case we get the results by applying Baer and Suzuki's results on groups having a non-trivial partition. A set $\beta=\left\{H_{1}, H_{2}, \ldots, H_{n}\right\}$ of subgroups $H_{i}$ $(i=1,2, \ldots, n)$ is said to be a partition of $G$ if every element $x \in G, x \neq 1$, belongs to one and only one subgroup $H_{i} \in \beta$. If $n=1$, the partition is said to be trivial.

Also we used the classification of Zassenhaus group of degree $q+1$. In fact, a Zassenhaus group of degree $q+1$ is a permutation group $G$ of degree $q+1$, in which the following hold:

(1) $G$ is doubly transitive on $q+1$ points.

(2) Any non-identity element has at most two fixed points.

(3) $G$ has no regular normal subgroups.

Note that, if $G$ acts on $\Omega$ and $K \leq G$ such that $K$ acts on $\Omega$ transitively and also for every $\alpha \in \Omega$ the stabilizer $K_{\alpha}$ is trivial, then we say $K$ is a regular subgroup of $G$.

For an integer $n$, we write $\pi(n)$ for the set of all prime divisors of $n$. We denote by $\pi(G)$, the set of all prime divisors of $|G|$. If $p$ is a prime number and $n$ is an integer, then we use the notation $n_{p}$ for $p$-part of $n$, that is, $n_{p}=p^{a}$, where $p^{a} \mid n$ and $p^{a+1} \nmid n$. If $\pi$ is a set of primes, by $G_{\pi}$ we mean a Hall $\pi$-subgroup of $G$ and in the particular case if $p$ is a prime, then $G_{p}$ denotes a Sylow $p$-subgroup of $G$. If $x \in G$, by index of $x$ in $G$ we mean the size of the conjugacy class containing $x$. All further unexplained notations are standard and are referred to [7], for example.

\section{Main Results}

The following result is the characterization of Zassenhaus groups of degree $q+1$, which follows from [9, Theorem 11.6].

Theorem 2.1. Suppose that $G$ is a Zassenhaus group of degree $q+1$ and order $(q+1) q d$. Then $q=p^{f}$ is a prime-power, and the followings are the only possibilities.

(a) $G=\mathrm{PGL}_{2}\left(p^{f}\right)$.

(b) $p$ is odd and $G=\mathrm{PSL}_{2}\left(p^{f}\right)$.

(c) $p$ is odd, $f$ is even and $G$ is a certain sharply triply transitive group of order $\left(q^{2}-1\right) q$ containing $\mathrm{PSL}_{2}\left(p^{f}\right)$ as its subgroup. 
(d) $p=2$ and $G$ is a Suzuki group $S z(q)$.

The following lemma describes the structure of a free centralizer which will be used frequently.

Lemma 2.2. ([5, Lemma 6]) Let $G$ be a group. If $C_{G}(x)$ is free, then either $C_{G}(x)$ is abelian or $C_{G}(x)=U_{p} \times \mathbf{Z}(G)_{p^{\prime}}$, where $U_{p}$ is a p-group for some prime $p$.

Lemma 2.3. ([2]) Let $G$ be a finite group. If the set of p-regular conjugacy class sizes of $G$ is exactly $\{1, m\}$, then $m=p^{a} q^{b}$, where $q$ is a prime distinct from $p$ and $a \geq 0, b \geq 0$. If $b=0$, then $G$ has an abelian p-complement. If $b \neq 0$, then $G=P Q \times A$, with $P \in \operatorname{Syl}_{p}(G), Q \in \operatorname{Syl}_{q}(G)$ and $A \leq \mathbf{Z}(G)$. Furthermore, if $a=0$, then $G=P \times Q \times A$.

Lemma 2.4. Let $G$ be a finite group with order $n p^{\alpha}$, where $(n, p)=1$ and $\alpha>0$. Assume $P$ and $R$ are two Sylow p-subgroups of $G$, such that $P \cap R$ has index $p$ in $P$. Then $\left|G: N_{G}(P \cap R)\right|=n / t$, where $t \mid n$ and $t>p$.

Proof. It is easy to see that $R P \subseteq N_{G}(P \cap R)$. Hence $\left|N_{G}(P \cap R)\right| \geq|P R|$. Now the result is obvious.

Lemma 2.5. Let $H \leq G$ such that $G=H \mathbf{Z}(G)$. Then $\operatorname{cs}(G)=\operatorname{cs}(H)$.

Proof. Since $G=H Z(G)$, this implies that $G=H C_{G}(r)$, for every $r \in G$. Therefore, for every $r \in G$, $\left|r^{G}\right|=\left|G: C_{G}(r)\right|=\left|H C_{G}(r): C_{G}(r)\right|=\left|H: H \cap C_{G}(r)\right|=\left|H: C_{H}(r)\right|=\left|r^{H}\right|$.

The following statement is taken from [8, Theorem A], which is the classification of $F$-groups.

Lemma 2.6. (Rebmann). Let $G$ be a nonabelian group. Then $G$ is an F-group if and only if it is one of the following groups:

(1) $G$ has a normal abelian subgroup of prime index.

(2) $G / \mathbf{Z}(G)$ is a Frobenius group with Frobenius kernel $K / \mathbf{Z}(G)$ and Frobenius complement $L / \mathbf{Z}(G)$, where $K$ and $L$ are abelian.

(3) $G / \mathbf{Z}(G)$ is a Frobenius group with Frobenius kernel $K / \mathbf{Z}(G)$ and Frobenius complement $L / \mathbf{Z}(G)$, such that $K=P \mathbf{Z}(G)$, where $P$ is a normal Sylow p-subgroup of $G$ for some prime $p \in \pi(G)$, $P$ is an $F$-group, $Z(P)=P \cap \mathbf{Z}(G)$ and $L=H \mathbf{Z}(G)$, where $H$ is an abelian $p^{\prime}$-subgroup of $G$.

(4) $G / \mathbf{Z}(G) \cong S_{4}$ and if $V / \mathbf{Z}(G)$ is the Klein four group in $G / \mathbf{Z}(G)$, then $V$ is nonabelian.

(5) $G=P \times A$, where $P$ is a nonabelian $F$-group of prime power order and $A$ is abelian.

(6) $G / \mathbf{Z}(G) \cong \mathrm{PSL}_{2}\left(p^{m}\right)$ or $\mathrm{PGL}_{2}\left(p^{m}\right)$ and $G^{\prime} \cong \mathrm{SL}_{2}\left(p^{m}\right)$, where $p$ is a prime and $p^{m}>3$.

(7) $G / \mathbf{Z}(G) \cong \mathrm{PSL}_{2}(9)$ or $\mathrm{PGL}_{2}(9)$ and $G^{\prime}$ is isomorphic to the Schur cover of $\mathrm{PSL}_{2}(9) \cong A_{6}$.

We note that except for the last two cases, $G$ is solvable.

Lemma 2.7. ([3,4]) If a solvable group $G$ has a non-trivial partition $\beta$, then one of the following conditions is satisfied:

(i) A component of $\beta$ is selfnormalized and $G$ is Frobenius.

(ii) $G \cong S_{4}$ and $\beta$ consists of maximal cyclic subgroups of $G$.

(iii) $G$ has a nilpotent normal subgroup $N$, which is a component of $\beta$ such that $|G: N|=p$ and all $x \in G \backslash N$ has order $p$.

(iv) $G$ is a p-group.

Lemma 2.8. ([12]) A finite non-solvable group $G$ has a non-trivial partition $\beta$ if and only if $G$ is isomorphic to one of the following groups:

(i) $G \cong S z(q)$, the Suzuki simple group.

(ii) $G \cong \mathrm{PSL}_{2}\left(p^{f}\right)$ or $\mathrm{PGL}_{2}\left(p^{f}\right)$, where $p$ is a prime, and $p^{f} \geq 4$. 
Since $\mathrm{PSL}_{2}(2)$ is not simple, the following theorem covers all finite simple groups $\mathrm{PSL}_{2}(q)$, for even $q$.

Theorem 2.9. Let $G$ be a finite group and $q>2$ even. If $\operatorname{cs}(G)=\operatorname{cs}\left(\mathrm{PSL}_{2}(q)\right)$, then $G \cong \mathrm{PSL}_{2}(q) \times A$, where $A$ is abelian.

Proof. It is known that if $q=2^{f}$, with $f \geq 2$, then

$$
\operatorname{cs}\left(\operatorname{PSL}_{2}(q)\right)=\left\{1, q(q-1), q^{2}-1, q(q+1)\right\}=\left\{1,2^{f}\left(2^{f}-1\right), 2^{2 f}-1,2^{f}\left(2^{f}+1\right)\right\} .
$$

Since there are no divisibilities among the nontrivial conjugacy class sizes of $\mathrm{PSL}_{2}\left(2^{f}\right)$, the centralizers of all non-trivial elements of $G$ are free. Hence, $G$ is an $F$-group. By using the classification of $F$-groups we have the following possibilities:

Case 1: $G$ has a normal abelian subgroup $H$ of prime index $p$.

If $H=\mathbf{Z}(G)$, then $G$ is abelian since $G / H$ is cyclic. Hence, $H-\mathbf{Z}(G)$ is nonempty. For $x \in H-\mathbf{Z}(G)$, we have $C_{G}(x)=H$ and then $\left|x^{G}\right|=p \notin \operatorname{cs}(G)$. So $G$ is not in this class.

Case 2: $G / \mathbf{Z}(G)$ is a Frobenius group with Frobenius kernel $K / \mathbf{Z}(G)$ and Frobenius complement $L / \mathbf{Z}(G)$, where $K$ and $L$ are abelian.

Pick $x \in L-\mathbf{Z}(G)$, we have $C_{G}(x)=L$. Hence $\left|x^{G}\right|=[G: L]=|K| /|\mathbf{Z}(G)|$. Similarly, pick $y \in K-\mathbf{Z}(G)$ we have $\left|y^{G}\right|=|L| /|\mathbf{Z}(G)|$. So $\operatorname{gcd}\left(\left|x^{G}\right|,\left|y^{G}\right|\right)=1$ which is not possible according to $\operatorname{cs}(G)$. Hence $G$ is not in this class.

Case 3: $G / \mathbf{Z}(G)$ is a Frobenius group with Frobenius kernel $K / \mathbf{Z}(G)$ and Frobenius complement $L / \mathbf{Z}(G)$ such that $K=P \mathbf{Z}(G)$, where $P$ is a normal Sylow $p$-subgroup of $G$ for some prime $p \in \pi(G)$, $P$ is an $F$-group, $\mathbf{Z}(P)=P \cap \mathbf{Z}(G)$ and $L=H \mathbf{Z}(G)$, where $H$ is an abelian $p^{\prime}$-subgroup of $G$.

It is clear that $G=K L$ and $K \cap L=\mathbf{Z}(G)$. Hence $|K|=|P||\mathbf{Z}(G)| /|P \cap \mathbf{Z}(G)|$ and $|G|=$ $|K L|=|K||L| /|\mathbf{Z}(G)|$. Pick $x \in L-\mathbf{Z}(G)$ we have $C_{G}(x)=L$. Thus $\left|x^{G}\right|=|G| /|L|=|K| /|\mathbf{Z}(G)|=$ $|P| /|P \cap \mathbf{Z}(G)|$ is a prime power which is not possible considering $\operatorname{cs}(G)$. So $G$ is not in this class.

Case 4: $G / \mathbf{Z}(G) \cong S_{4}$ and if $V / \mathbf{Z}(G)$ is the Klein four group in $G / \mathbf{Z}(G)$, then $V$ is nonabelian.

For each $x \in G-\mathbf{Z}(G)$ we have $\mathbf{Z}(G) \leq C_{G}(x)$ and $\left[G: C_{G}(x)\right]|[G: \mathbf{Z}(G)]=| S_{4} \mid=2^{3} \cdot 3$. This shows that all primes dividing conjugacy class sizes are either 2 or 3 , which contradicts the fact that $2^{f}-1,2^{f}+1$ and $2^{f}$ are pairwise coprime. Hence $G$ is not in this class.

Case 5: $G=P \times A$, where $P$ is a nonabelian $F$-group of prime power order and $A$ is abelian.

It is clear that the conjugacy class sizes are all prime powers. Therefore, $G$ is not in this class.

Case 6: $G / \mathbf{Z}(G) \cong \mathrm{PSL}_{2}\left(p^{m}\right)$ or $\mathrm{PGL}_{2}\left(p^{m}\right)$ and $G^{\prime} \cong \mathrm{SL}_{2}\left(p^{m}\right)$, where $p$ is a prime and $p^{m}>3$.

If $p^{m}=5$, then $\operatorname{cs}\left(G^{\prime}\right)=\{1,12,20,30\}$ and $|G / \mathbf{Z}(G)|=60$ or $120=2^{3} \cdot 15$. Since $\left|x^{G}\right|=[G$ : $\left.C_{G}(x)\right] \mid[G: \mathbf{Z}(G)]$ for all $x \in G$, we have $2^{f} \mid 2^{3}$ and $2^{f} \pm 1 \mid 15$. Since $f \geq 2$, it implies $f=2$. So $\operatorname{cs}(G)=\{1,12,15,20\}$ which contains no multiples of $30 \in \operatorname{cs}\left(G^{\prime}\right)$. So $G$ does not satisfy this case.

Suppose that $p^{m} \geq 7$ is odd and $p^{m} \equiv \nu(\bmod 4)$, where $\nu \in\{-1,1\}$. We have $\left|\mathrm{SL}_{2}\left(p^{m}\right)\right|=$ $\left|\mathrm{PGL}_{2}\left(p^{m}\right)\right|=p^{m}\left(p^{2 m}-1\right)$, and $\operatorname{cs}\left(\mathrm{SL}_{2}\left(p^{m}\right)\right)=\left\{1,\left(p^{2 m}-1\right) / 2, p^{m}\left(p^{m}-1\right), p^{m}\left(p^{m}+1\right)\right\}$.

Since each $r \in \operatorname{cs}\left(G^{\prime}\right)$ is a divisor of some element in $\operatorname{cs}(G)$ and $p^{m}\left(p^{m}+\nu\right)$ is even, we conclude that $p^{m}\left(p^{m}+\nu\right)$ is a divisor of either $2^{f}\left(2^{f}-1\right)$ or $2^{f}\left(2^{f}+1\right)$. Since $p^{m}\left(p^{m}+\nu\right) / 2$ is odd, we infer that $p^{m}\left(p^{m}+\nu\right) / 2$ is a divisor of either $2^{f}-1$ or $2^{f}+1$. Thus $p^{m}\left(p^{m}-1\right) / 2 \leq p^{m}\left(p^{m}+\nu\right) / 2 \leq 2^{f}+1$. By $p^{m} \geq 7$, we have $2\left(p^{m}+1\right)<p^{m}\left(p^{m}-1\right) / 2-2$. So $2\left(p^{m}+1\right)<2^{f}-1$. Therefore, $p^{m}\left(p^{2 m}-1\right)<2^{2 f}-1$. On the other hand, for each $x \in G$, we have $\left|x^{G}\right|=\left[G: C_{G}(x)\right] \mid[G: \mathbf{Z}(G)]=p^{m}\left(p^{2 m}-1\right)$. Hence $s \leq p^{m}\left(p^{2 m}-1\right)$ for all $s \in \operatorname{cs}(G)$, which is a contradiction, by the above argument. Thus $G$ does not satisfy this case.

Now we suppose that $p^{m}$ is even. So $G / \mathbf{Z}(G) \cong G^{\prime} \cong \mathrm{PSL}_{2}\left(2^{m}\right)$, hence

$$
\operatorname{cs}\left(G^{\prime}\right)=\left\{1,2^{m}\left(2^{m}-1\right), 2^{2 m}-1,2^{m}\left(2^{m}+1\right)\right\}
$$

and $|G / \mathbf{Z}(G)|=2^{m}\left(2^{2 m}-1\right)$. Since every $r \in \operatorname{cs}\left(G^{\prime}\right)$ is a divisor of some number in $\operatorname{cs}(G)$, we have $2^{m} \mid 2^{f}$. Since all $r \in \operatorname{cs}(G)$ are divisors of $|G / \mathbf{Z}(G)|$, we have $2^{f} \mid 2^{m}$. Thus $2^{f}=2^{m}$ and so $m=f$. 
It is clear that $G^{\prime} \cap \mathbf{Z}(G)=\{1\}$ and $\left|G / G^{\prime}\right|=|\mathbf{Z}(G)|$. Hence $G=G^{\prime} \mathbf{Z}(G) \cong G^{\prime} \times \mathbf{Z}(G)$, which is the result.

Case 7: $G / \mathbf{Z}(G) \cong \mathrm{PSL}_{2}(9)$ or $\mathrm{PGL}_{2}(9)$ and $G^{\prime}$ is isomorphic to a Schur cover of $\mathrm{PSL}_{2}(9) \cong A_{6}$.

We have $\left|\mathrm{PSL}_{2}(9)\right|=360$, and $\left|\mathrm{PGL}_{2}(9)\right|=720$. Using GAP, we have $90 \in \operatorname{cs}\left(G^{\prime}\right)$ for any Schur cover of $A_{6}$.

Since $720=2^{4} \cdot 45$, by considering $\operatorname{cs}(G)$ and the fact that $\left|x^{G}\right| \mid[G: \mathbf{Z}(G)]$ for all $x \in G$, we obtain $2^{f} \mid 2^{4}$ and $2^{f} \pm 1 \mid 45$. By checking directly with $f=2,3,4$, we have $f=2$. So $\operatorname{cs}(G)=\{1,12,15,20\}$ which contains no multiple of $90 \in \operatorname{cs}\left(G^{\prime}\right)$. Thus $G$ does not satisfy this case.

Note that $\mathrm{PSL}_{2}(5) \cong \mathrm{PSL}_{2}(4)$ and $\mathrm{PSL}_{2}(3) \cong A_{4}$ is not simple. The following theorem covers all finite simple groups $\mathrm{PSL}_{2}(q)$, for odd number $q$.

Theorem 2.10. Let $G$ be a group such that $\operatorname{cs}(G)=\operatorname{cs}\left(\mathrm{PSL}_{2}(q)\right)$, where $q=p^{f} \geq 7$ and $p$ is an odd prime. Then $G / \mathbf{Z}(G) \cong \operatorname{PSL}_{2}(q)$.

The proof of Theorem 2.10 follows from a series of Lemmas and Remarks $2.11-2.18$. In the following we assume that $G$ satisfies the hypothesis of Theorem 2.10 .

Remark 2.11. Since $q \equiv \nu(\bmod 4)$, where $\nu \in\{-1,+1\}$, we have

$$
\operatorname{cs}\left(\operatorname{PSL}_{2}(q)\right)=\left\{1,\left(q^{2}-1\right) / 2, q(q+\nu) / 2, q(q-\nu), q(q+\nu)\right\} .
$$

By a well-known result in [6] we may assume that $\pi(G)=\pi\left(q\left(q^{2}-1\right)\right)$. Since $q \equiv \nu(\bmod 4)$, $(q+\nu) / 2$ is odd and $(q-\nu) / 2$ is even. So $(q+\nu) / 2, q-\nu$ and $q$ are pairwise coprime. Hence there is no prime $t \in \pi(G)$ such that $t \mid a$ for all $a \in \operatorname{cs}(G)-\{1\}$.

Lemma 2.12. For each $t \in \pi(q(q+\nu) / 2), G_{t}$ is abelian.

Proof. First we claim for each $t \in \pi(q(q+\nu) / 2)$, we have $\mathbf{Z}\left(G_{t}\right)-\mathbf{Z}(G)$ is nonempty. Let $x$ be a noncentral element of $G$ such that $G_{t} \leq C_{G}(x)$. Therefore $\left|x^{G}\right| \in\left\{\left(q^{2}-1\right) / 2, q(q-\nu)\right\}$, and so $C_{G}(x)$ is free. By Lemma 2.2, $C_{G}(x)$ is either abelian or isomorphic to $U_{s} \times \mathbf{Z}(G)_{s^{\prime}}$, where $U_{s}$ is an $s$-group for some prime divisor $s$ of $|G|$.

Suppose that $C_{G}(x)$ is not abelian. So $C_{G}(x)=U_{s} \times \mathbf{Z}(G)_{s^{\prime}}$, where $U_{s}$ is an $s$-group and $s \in \pi(G)$. Note that $G_{t} \leq C_{G}(x)$. If $s \neq t$, then $G_{t} \leq \mathbf{Z}(G)_{s^{\prime}} \leq \mathbf{Z}(G)$ which is impossible, by the fact that $t$ is a divisor of some conjugacy class size of $G$. Hence $s=t$ and $C_{G}(x)=G_{t} \times \mathbf{Z}(G)_{t^{\prime}}$. So we can assume that $x$ is a $t$-element and we conclude that $x \in \mathbf{Z}\left(G_{t}\right)-\mathbf{Z}(G)$.

Now we may assume $C_{G}(x)$ is abelian. Since $G_{t} \leq C_{G}(x)$, it is clear that $G_{t}$ is abelian and so $\mathbf{Z}\left(G_{t}\right)=G_{t}$. If $G_{t} \leq \mathbf{Z}(G)$, then $t$ does not divide any conjugacy class sizes, a contradiction. So our claim is proved.

Let $N=q\left(q^{2}-1\right) / 2$. For each $t \in \pi(q(q+\nu) / 2)$, we shall show that $G_{t}$ is abelian by contradiction. We assume that $G_{t}$ is not abelian. For every $y \in \mathbf{Z}\left(G_{t}\right)-\mathbf{Z}(G)$, we have $C_{G}(y)=G_{t} \times \mathbf{Z}(G)_{t^{\prime}}$. Hence $C_{G}\left(\mathbf{Z}\left(G_{t}\right)\right)=\cap_{y \in \mathbf{Z}\left(G_{t}\right)} C_{G}(y)=G_{t} \times \mathbf{Z}(G)_{t^{\prime}}$. So there is no non-central $t^{\prime}$-element centralizing $\mathbf{Z}\left(G_{t}\right)$.

Since $G_{t}$ is not abelian, there is $u \in G_{t}-\mathbf{Z}\left(G_{t}\right)$. So $\mathbf{Z}\left(G_{t}\right) \lesseqgtr C_{G}(u)_{t}$. By $\operatorname{cs}(G)$, we infer that $\left|G_{t}\right|=N_{t}\left|C_{G}(u)_{t}\right|>N_{t}\left|\mathbf{Z}\left(G_{t}\right)\right|$, where either $N_{t}=q$ or $N_{t}=((q+\nu) / 2)_{t}$.

If $t \in \pi((q+\nu) / 2)$, then let $s=p$; otherwise if $t=p$, then choose $s \in \pi((q+\nu) / 2)$. Pick $x \in \mathbf{Z}\left(G_{s}\right)-\mathbf{Z}(G)$. We have $\left|G: C_{G}(x)\right| \in\left\{q(q-\nu),\left(q^{2}-1\right) / 2\right\}$. Without loss of generality we may assume $C_{G}(x)_{t} \lesseqgtr G_{t}$. Note that $\left|G_{t}: C_{G}(x)_{t}\right|=N_{t}$ and hence $\left|C_{G}(x)_{t}\right|>\left|\mathbf{Z}\left(G_{t}\right)\right|$, which implies that there is $y \in C_{G}(x)_{t}-\mathbf{Z}\left(G_{t}\right) \subset G_{t}$. But we know that $C_{G}(x)$ is free and so either $C_{G}(x)$ is abelian or $C_{G}(x)=G_{s} \times \mathbf{Z}(G)_{s^{\prime}}$. By the fact that $\left|C_{G}(x)_{t}\right|>\left|\mathbf{Z}\left(G_{t}\right)\right|$, we deduce that $C_{G}(x)$ is abelain.

Note that $\mathbf{Z}\left(G_{t}\right) \lesseqgtr C_{G}(y)$. By the fact that $C_{G}(x)$ is abelian, we have $C_{G}(x) \leq C_{G}(y)$. Since $C_{G}(x)$ is free and $C_{G}(y) \lesseqgtr G$, we have $C_{G}(x)=C_{G}(y)$. Hence $\mathbf{Z}\left(G_{t}\right) \leq C_{G}(x)$ and so $x$ centralizes $\mathbf{Z}\left(G_{t}\right)$. Since $x$ is a $t^{\prime}$-element, it contradicts the above argument. Thus, $G_{t}$ is abelian. 
Remark 2.13. By Lemmas 2.12 and 2.2 , for every $x \in G$ such that $\left|x^{G}\right| \in\left\{\left(q^{2}-1\right) / 2, q(q-\nu)\right\}, C_{G}(x)$ is abelian.

Lemma 2.14. Let $g \in G-\mathbf{Z}(G)$. The following hold.

(i) If $\left|g^{G}\right| \in\{q(q+\nu), q(q+\nu) / 2\}$, then $C_{G}(g) / \mathbf{Z}(G)$ is a $\pi(q-\nu)$-group.

(ii) If $\left|g^{G}\right|=q(q-\nu)$, then $C_{G}(g) / \mathbf{Z}(G)$ is a $\pi((q+\nu) / 2)$-group.

(iii) If $\left|g^{G}\right|=\left(q^{2}-1\right) / 2$, then $C_{G}(g) / \mathbf{Z}(G)$ is a p-group.

Proof. (i) Assume $g \in G$ such that $\left|g^{G}\right| \in\{q(q+\nu), q(q+\nu) / 2\}$. Let $t \in \pi(q(q+\nu) / 2)$. Write $g=g_{t} g_{t^{\prime}}=g_{t^{\prime}} g_{t}$, where $g_{t}$ and $g_{t^{\prime}}$ are $t$-part and $t^{\prime}$-part of $g$, respectively (it means $g_{t}$ is a $t$-element and $g_{t^{\prime}}$ is a $t^{\prime}$-element). We know $C_{G}(g)=C_{G}\left(g_{t}\right) \cap C_{G}\left(g_{t^{\prime}}\right)$. If $g_{t} \notin \mathbf{Z}(G)$, then $G_{t} \leq C_{G}\left(g_{t}\right)$ and $\left|g_{t}^{G}\right|=\left|G: C_{G}\left(g_{t}\right)\right|$ divides $\left|G: C_{G}(g)\right|=\left|g^{G}\right|$ which is impossible since by Lemma 2.12 we have $\left|g_{t}^{G}\right| \in$ $\left\{\left(q^{2}-1\right) / 2, q(q-\nu)\right\}$. Hence $g_{t} \in \mathbf{Z}(G)$. Now let $x \in C_{G}(g)$, be a $t$-element, for some $t \in \pi(q(q+\nu) / 2)$. So $C_{G}\left(g_{t^{\prime}} x\right)=C_{G}(x) \cap C_{G}\left(g_{t^{\prime}}\right)$. Hence $\left|g_{t^{\prime}}^{G}\right|||\left(x g_{t^{\prime}}\right)^{G} \mid$ and so $\left|\left(x g_{t^{\prime}}\right)^{G}\right| \in\{q(q+\nu), q(q+\nu) / 2\}$. Therefore similar to the above discussion we conclude $x$ is central. So $C_{G}(g) / \mathbf{Z}(G)$ is a $\pi(q-\nu)$-group. Thus part (i) is proved.

(ii) Let $x$ be an element whose index is $q(q-\nu)$. By Remark $2.13, C_{G}(x)$ is abelian. If there is a $p$-element $y \in C_{G}(x)-\mathbf{Z}(G)$, then $C_{G}(x) \leq C_{G}(y)$, and so $\left|y^{G}\right|$ divides $\left|x^{G}\right|$, which contradicts $\left|y^{G}\right|=\left(q^{2}-1\right) / 2$.

Now we assume that there is a $t$-element $y \in C_{G}(x)-\mathbf{Z}(G)$, for some $t \in \pi(q-\nu)$. By Remark 2.13, $C_{G}(x)$ is abelian and free, it implies that $C_{G}(y)=C_{G}(x)$. From the set of conjugacy class sizes and part (i), it is clear that there exists a $\pi(q-\nu)$-element $z \in C_{G}(y)$ whose index is $q(q+\nu) / 2$ and centralizes a $G_{s}$, for all $s \in \pi(q-\nu)$. Since $C_{G}(y)$ is abelian, we have $C_{G}(y) \leq C_{G}(z)$. So $\left|z^{G}\right|=\left|G: C_{G}(z)\right|$ divides $\left|G: C_{G}(y)\right|=\left|y^{G}\right|=q(q-\nu)$, a contradiction. Therefore $C_{G}(x) / \mathbf{Z}(G)$ is a $\pi((q+\nu) / 2)$-group.

(iii) Let $x \in G$ have index $\left(q^{2}-1\right) / 2$. If there is a $t$-element $y \in C_{G}(x)-\mathbf{Z}(G)$ for some $t \in \pi((q+\nu) / 2)$, then by Lemma $2.12 C_{G}(x) \leq C_{G}(y)$, and so $\left|y^{G}\right|$ divides $\left|x^{G}\right|$, which is impossible, since $\left|y^{G}\right|=q(q-\nu)$.

Now we assume that there is a $t$-element $y \in C_{G}(x)-\mathbf{Z}(G)$ for some $t \in \pi(q-\nu)$. Since $C_{G}(x)$ is free, it implies that $C_{G}(y)=C_{G}(x)$. Again by part (i), there exists a $\pi(q-\nu)$-element $z \in C_{G}(y)$ of index $q(q+\nu) / 2$. Thus $\left|z^{G}\right|=\left|G: C_{G}(z)\right|$ divides $\left|G: C_{G}(y)\right|=\left|y^{G}\right|=\left(q^{2}-1\right) / 2$, a contradiction. Thus $C_{G}(x) / \mathbf{Z}(G)$ is a $p$-group.

In the following, we set $\bar{G}=G / \mathbf{Z}(G)$ and $\bar{x}=x \mathbf{Z}(G) \in \bar{G}$ for every $x \in G$.

Lemma 2.15. The following hold.

(i) $|\bar{G}|=q\left(q^{2}-1\right) / 2$,

(ii) Let $\bar{x}$ and $\bar{y}$ are an s-element and a t-element in $\bar{G}$, respectively, when either $s \in \pi(q-\nu)$ and $t \in \pi((q+\nu) / 2), s=p$ and $t \in \pi(q-\nu)$, or $s=p$ and $t \in \pi((q+\nu) / 2)$. Then $[\bar{x}, \bar{y}] \neq 1$.

(iii) $G$ has Hall $\pi(q-\nu)$-subgroups and Hall $\pi((q+\nu) / 2)$-subgroups.

Proof. (i) By Lemma 2.14, there exists $x \in G$ such that $\left|G: C_{G}(x)\right|_{p}=q$, and $\left|C_{G}(x) / \mathbf{Z}(G)\right|_{p}=1$. Hence, $|G: \mathbf{Z}(G)|_{p}=q$. Similarly we conclude that $|G: \mathbf{Z}(G)|_{t}=\left(\left(q^{2}-1\right) / 2\right)_{t}$, for every prime $t \in \pi\left(\left(q^{2}-1\right) / 2\right)$. Therefore, $|\bar{G}|=q\left(q^{2}-1\right) / 2$.

(ii) Suppose that there are an $s$-element $\bar{x}$ and a $t$-element $\bar{y}$ in $\bar{G}$ such that $\bar{x} \bar{y}=\bar{y} \bar{x}$, where $s \in \pi(q-\nu), t \in \pi((q+\nu) / 2)$. Then we may assume $x$ and $y$ are an $s$-element and a $t$-element, respectively. We have $[x, y] \in \mathbf{Z}(G)$. If $o(x)=k$, then $\left[x^{k}, y\right]=\left[x, y^{k}\right]=1$, so $x \in C_{G}\left(y^{k}\right)=C_{G}(y)$ since $\operatorname{gcd}(k, t)=1$. This is a contradiction by Lemma 2.14 (ii). We apply the same argument for the pair $(s, t)$ where either $s=p$ and $t \in \pi((q+\nu) / 2)$, or $s=p$ and $t \in \pi(q-\nu)$, then we get another contradiction. 
(iii) By Lemma 2.14 (i) and (ii), $\bar{G}$ has Hall $\pi(q-\nu)$-subgroups and Hall $\pi((q+\nu) / 2)$-subgroups. Let $K / \mathbf{Z}(G)=C_{G}(y) / \mathbf{Z}(G)$ be a Hall $\pi(q-\nu)$-subgroup of $\bar{G}$, for some $y \in G$. So $K /\left(\mathbf{Z}(G)_{\pi(q-\nu)^{\prime}}\right)$ is a $\pi(q-\nu)$-group. By Schur-Zassenhaus Theorem, there exists $H \leq G$ such that $K=H \ltimes \mathbf{Z}(G)_{\pi(q-\nu)^{\prime}}=$ $H \times \mathbf{Z}(G)_{\pi(q-\nu)^{\prime}}$ and $H$ is a $\pi(q-\nu)$-group. It is easy to see that $H$ is a Hall $\pi(q-\nu)$-subgroup of $G$. We can discuss similarly to deduce that $G$ has Hall $\pi((q+\nu) / 2)$-subgroups.

Lemma 2.16. Let $\pi(q-\nu)-\{2\} \neq \emptyset$. Assume that $H$ is a Hall $\pi(q-\nu)$-subgroup of $G$. Then one of the following holds.

(i) $H=Q \times A$, where $Q$ is a Sylow 2-subgroup of $H$ and $A$ is a normal abelian 2-complement of $H$,

(ii) $H=Q \ltimes A$, where $Q$ is a Sylow 2-subgroup of $H$ and $A$ is an abelian 2-complement of $H$. Furthermore, $\left|x^{G}\right|=q(q+\nu)$ for every $2^{\prime}$-element $x \in H-\mathbf{Z}(G)$, and $C_{G}(x)$ is abelian for every $x \in H$ with $\left|x^{G}\right|=q(q+\nu)$.

Proof. Pick $y \in G$ such that $\left|y^{G}\right|=q(q+\nu) / 2$. As we discussed in the proof of 2.15 (iii), $C_{G}(y)=$ $H \times \mathbf{Z}(G)_{\pi(q-\nu)^{\prime}}$ where $H$ is the Hall $\pi(q-\nu)$-subgroup of $G$. By the maximality of $C_{G}(y)$, we may assume $y$ is a $t$-element, for some prime $t$. Let $x \in C_{G}(y)$ be a $t^{\prime}$-element. Note that there exists such an element, since $|\pi(q-\nu)| \geq 2$. Since $C_{C_{G}(y)}(x)=C_{G}(x y)=C_{G}(x) \cap C_{G}(y) \leq C_{G}(y)$ and from $\operatorname{cs}(G)$, the index of $x$ in $C_{G}(y)$ is either 1 or 2 . Since $H \unlhd C_{G}(y)$, it follows that the index of every $t^{\prime}$-elements of $H$ is 1 or 2 .

First we assume the indices of all $t^{\prime}$-elements of $H$ are 1 . So $H$ has a central $t$-complement. If $t \neq 2$, then $H=T \times A$, where $T$ is a Sylow $t$-subgroup of $G$ and $A$ is an abelian 2-complement of $H$. This implies that $C_{G}(x)$ contains a Sylow 2-subgroup of $G$ for all $x \in H$, and thus, $\left|x^{G}\right|=q(q+\nu) / 2$, which contradicts Lemma 2.14. So $t=2$ and we have $H=Q \times A$, where $Q$ is a Sylow 2-subgroup of $H$ and $A$ is an abelian 2-complement of $H$, as we claimed in (i).

Secondly, we assume the set of indices of $t^{\prime}$-elements of $H$ equals $\{1,2\}$. By Lemma 2.3, we consider the following two cases of $t$ :

(a) If $t \neq 2$, then $H=T \times Q \times A^{\prime}$, where $T \in \operatorname{Syl}_{t}(H), Q \in \operatorname{Syl}_{2}(H)$ and $A^{\prime} \leq \mathbf{Z}(H)$. From $\operatorname{cs}(G)$, every $2^{\prime}$-element $z \in H$ has $\left|z^{G}\right|=q(q+\nu) / 2=\left|y^{G}\right|$. Thus, there must exist a 2-element $x$ such that $\left|C_{G}(x)\right|=\left|C_{G}(y)\right| / 2$. For all 2'-elements $z \in C_{G}(x)$, by the minimality of $C_{G}(x)$ we have $C_{G}(x z)=C_{G}(x) \cap C_{G}(z)=C_{G}(x) \leq C_{G}(z)$, which implies $z \in \mathbf{Z}\left(C_{G}(x)\right)$. So by setting $A=T \times A^{\prime}$, we have $H=Q \times A$ where $A$ is abelian, as we claimed in (i).

(b) If $t=2$, by Lemma 2.3, $H$ has abelian 2-complements. Here we may assume that all non-central $2^{\prime}$-elements $x \in H$ have $\left|x^{G}\right|=q(q+\nu)$, since if there exists a $2^{\prime}$-element $x$ such that $\left|C_{G}(x)\right|=\left|C_{G}(y)\right|$, then similar to case (a), we deduce (i) holds.

First assume that $x \in H$ is a $2^{\prime}$-elements such that $\left|x^{G}\right|=q(q+\nu)$. Then by the minimality of $C_{G}(x)$, we have $C_{G}(x)_{2} \leq \mathbf{Z}\left(C_{G}(x)\right)$. Since $H$ has abelian 2-complements, $C_{G}(x)$ is abelian. Now let $x$ be a 2element such that $\left|x^{G}\right|=q(q+\nu)$. Let $z \in C_{G}(x)$ be a non-central $2^{\prime}$-element. Then $C_{G}(x) \leq C_{G}(z)$ and so $C_{G}(x)=C_{G}(z)$, by the fact that $\left|C_{G}(z)\right|=\left|C_{G}(y)\right| / 2=\left|C_{G}(x)\right|$, for every non-central 2'-element $z$. Since $C_{G}(z)$ is abelian, $C_{G}(x)$ is also abelian. Hence for every $x$ with $\left|x^{G}\right|=q(q+\nu), C_{G}(x)$ is abelian, as we claimed in (ii).

Let $\left|x^{G}\right|=q(q+\nu)$, for some $x \in G$. Then $C_{G}(x)_{\pi(q-\nu)} \leq H$, for some Hall $\pi(q-\nu)$-subgroup $H$ and $\left|H: C_{G}(x)_{\pi(q-\nu)}\right|=2$. Thus $C_{G}(x)_{\pi(q-\nu)} \unlhd H$. We also know that $C_{G}(x)_{\pi(q-\nu)}$ is abelian and so $C_{G}(x)_{\pi(q-\nu)}$ has a normal 2-complement. Hence $H$ has a normal 2-complement as well. Therefore $H=Q \ltimes A$, where $A$ is the 2-complement of $H$ and $Q$ is a Sylow 2-subgroup of $H$ and so (ii) holds.

Lemma 2.17. Let $\pi(q-\nu)-\{2\} \neq \emptyset$. Then $\bar{G} \cong \operatorname{PSL}(2, q)$.

Proof. We shall show that $\bar{G}$ has a non-trivial partition. By Lemma 2.16, we have the following cases:

First, assume $H=Q \times A$, where $H$ is a Hall $\pi(q-\nu)$-subgroup of $G, Q$ is a Sylow 2-subgroup of $G$, and $A$ is an abelian 2-complement of $H$. So by well-known Wielandt's Theorem (see [10, 9.1.10]), all Hall $\pi(q-\nu)$-subgroups of $G$ are conjugate. 
Let $\beta=\left\{C_{G}(x) / \mathbf{Z}(G): x\right.$ is a non-central element of $G$ such that $C_{G}(x)$ is maximal in the lattice of centralizers of $G$ \}. Since the elements in $\beta$ are either Hall $\pi(q-\nu)$-subgroups, Hall $\pi(q+\nu) / 2$ subgroups or Sylow $p$-subgroups of $\bar{G}$, we can see that union of elements of $\beta$ is a cover for $\bar{G}$. Let $z \in C_{G}(x) \cap C_{G}(y)-\mathbf{Z}(G)$. By Lemma 2.14 and the maximality of $C_{G}(x), C_{G}(y)$, we have $\left|x^{G}\right|=\left|y^{G}\right|$. First, assume $\left|x^{G}\right|=\left|y^{G}\right| \in\left\{\left(q^{2}-1\right) / 2, q(q-\nu)\right\}$. Since $C_{G}(x)$ is abelian by Remark 2.13, we have $C_{G}(x) \leq C_{G}(z)$ and also by the maximality of $C_{G}(x)$, we conclude that $C_{G}(x)=C_{G}(z)$. Similarly we have $C_{G}(y)=C_{G}(z)$ and so $C_{G}(x)=C_{G}(y)$.

Now let $\left|x^{G}\right|=\left|y^{G}\right|=q(q+\nu) / 2$. As we discussed in the proof of Lemma 2.15 (iii) we may assume $C_{G}(x)=H \times \mathbf{Z}(G)_{\pi(q-\nu)^{\prime}}$ and hence $C_{G}(y)=H^{g} \times \mathbf{Z}(G)_{\pi(q-\nu)^{\prime}}$, for some $g \in G$.

Since $A$ and $A^{g}$ are central in $C_{G}(x)$ and $C_{G}(y)$, respectively, we have $C_{G}(z)$ contains $A$ and $A^{g}$ as its subgroups. Note that, considering the structure of $C_{G}(x)$, we may assume $z$ is a non-central $\pi(q-\nu)$ element. So $\left|z^{G}\right| \in\{q(q+\nu), q(q+\nu) / 2\}$, which implies that $C_{G}(z)_{\pi(q-\nu)} \leq H^{k}$, for some $k \in G$, by using Lemma 2.14. Thus $A$ is normal in $C_{G}(z)$, which implies that $A=A^{g}$. Since $\pi(q-\nu)-\{2\} \neq \emptyset$, we have $\left\langle Q, Q^{g}\right\rangle \leq C_{G}(A)=C_{G}(x)$. By the fact that $Q$ is normal in $C_{G}(x)$, we have $Q=Q^{g}$. Thus $C_{G}(x)=C_{G}(y)=H \times \mathbf{Z}(G)_{\pi(q-\nu)^{\prime}}$. Therefore $\beta$ is a partition for $\bar{G}$.

Secondly, assume $H=Q \ltimes A$, where $Q$ is a Sylow 2-subgroup of $H$ and $A$ is a normal abelian 2-complement of $H, C_{G}(x)$ is abelian for every $x \in G$ with $\left|x^{G}\right|=q(q+\nu)$, and $\left|y^{G}\right|=q(q+\nu)$ for every $2^{\prime}$-element $y \in H-\mathbf{Z}(G)$.

Let $\beta=\left\{C_{G}(x) / \mathbf{Z}(G): x\right.$ is a non-central element of $G$ such that $C_{G}(x)$ is minimal in the lattice of centralizers of $G\}$. We claim that union of elements of $\beta$ is a cover for $\bar{G}$. It is obvious that all of the elements of $\bar{G}$, beside 2-elements whose indices are $q(q+\nu) / 2$, appear in the components of $\beta$. Assume $x$ is a 2-element whose index in $G$ is $q(q+\nu) / 2$. Then $H \leq C_{G}(x)$, for some Hall $\pi(q-\nu)$-subgroup $H$ of $G$. Thus $x \in \mathbf{Z}(H)$. To fulfil the claim that the union of components of $\beta$ covers $\bar{G}$, it suffices to find $y \in H$ such that $\left|C_{G}(y)\right|=\left|C_{G}(x)\right| / 2$, then $x \in C_{G}(y)$.

Let $z \in C_{G}(x) \cap C_{G}(y)-\mathbf{Z}(G)$. We know that $\left|x^{G}\right|=\left|y^{G}\right|$, by Lemma 2.14. If $\left|x^{G}\right|=\left|y^{G}\right| \in$ $\left\{\left(q^{2}-1\right) / 2, q(q-\nu)\right\}$, then similarly to the first case we get $C_{G}(x)=C_{G}(y)$. If $\left|x^{G}\right|=\left|y^{G}\right|=q(q+\nu)$, then, by the fact that $C_{G}(x)$ and $C_{G}(y)$ are abelian, we have $C_{G}(x) \leq C_{G}(z)$ and $C_{G}(y) \leq C_{G}(z)$. Thus either $C_{G}(x)=C_{G}(z)=C_{G}(y)$, or $C_{G}(z)=H \times \mathbf{Z}(G)_{\pi(q-\nu)^{\prime}}$, for some Hall $\pi(q-\nu)$-subgroup $H$ of $G$. The former case is what we wanted to prove, so we may assume the later case holds. Since $H$ has a unique 2-complement, we deduce that $C_{G}(x), C_{G}(y)$ and $C_{G}(z)$ have the same 2-complement. Let $C_{G}(x)=A \times Q_{1} \times \mathbf{Z}(G)_{\pi(q-\nu)^{\prime}}$ and $C_{G}(y)=A \times Q_{2} \times \mathbf{Z}(G)_{\pi(q-\nu)^{\prime}}$, where $A \times \mathbf{Z}(G)_{\pi(q-\nu)^{\prime}}$ is the 2-complement of $C_{G}(x)$ and $C_{G}(y)$, and $Q_{1}$ and $Q_{2}$ are Sylow 2-subgroups of $C_{G}(x)$ and $C_{G}(y)$, respectively. For $u \in A-\mathbf{Z}(G)$ we have $\left|C_{G}(u)\right|=\left|C_{G}(x)\right|=\left|C_{G}(y)\right|$. Also $C_{G}(x) \leq C_{G}(u)$ and $C_{G}(y) \leq C_{G}(u)$. Hence $C_{G}(u)=C_{G}(x)=C_{G}(y)$. Therefore $\beta$ is a partition for $\bar{G}$.

So by Lemmas 2.7 and 2.8 , one of the following cases occurs:

Case 1. Similar to the argument we had in Theorem 2.9, we have $\bar{G}$ is not a $p$-group and also it is not isomorphic to $S_{4}$.

Case 2. Let $\bar{G}$ has a normal subgroup, say $N$, such that $|\bar{G}: N|=r$, where $r$ is a prime number and $N$ is nilpotent.

In this case $N$ is one of the components of $\beta$ and so $|N| \in\{q,(q-\nu),(q-\nu) / 2,(q+\nu) / 2\}$, which is not possible since $|\bar{G} / N|$ is prime.

Case 3. $\bar{G}$ is a Frobenious group.

Since the Frobenius kernel of $\bar{G}$ is nilpotent, we have $K$, the Frobenius kernel of $\bar{G}$, is either a Sylow $p$-subgroup of $\bar{G}$, a Hall $\pi(q-\nu)$-subgroup of $\bar{G}$, or a Hall $\pi((q+\nu) / 2)$-subgroup of $\bar{G}$, by Lemma 2.15 (ii). If $H$ is a Frobenius complement of $\bar{G}$, then $(|K|,|H|) \in\{(q-\nu, q(q+\nu) / 2),((q+\nu) / 2, q(q-$ $\left.\nu)),\left(q,\left(q^{2}-1\right) / 2\right)\right\}$, which is contradicting $|H||| K \mid-1$.

Case 4. $\bar{G}$ is isomorphic to $S z\left(2^{h}\right)$, for some odd integer $h \geq 3$.

Then $|\bar{G}|=\left|S z\left(2^{h}\right)\right|=2^{2 h}\left(2^{2 h}+1\right)\left(2^{h}-1\right)$. It is well-known that 2-elements in $S z\left(2^{h}\right)$ does not commute with any $t$-elements, for prime $t$ different from 2 . Hence $q-\nu=2^{2 h}$, which contradicts the hypothesis of this Lemma. 
Case 6. $\bar{G}$ is isomorphic to $\mathrm{PGL}_{2}\left(r^{h}\right)$, where $r^{h} \geq 4$.

Since $\left|\operatorname{cs}\left(\mathrm{PGL}_{2}\left(r^{h}\right)\right)\right|=6$, when $r^{h}$ is odd, we may assume $r=2$. Hence $2^{h} \mid q-\nu$ and also $q \mid 2^{h} \pm 1$. Hence $2^{h}-1 \leq q \leq 2^{h}+1$ and so $q=2^{h} \pm 1$. But we know that $(q+\nu) / 2$ is odd and $\pi(q-\nu) \neq\{2\}$, a contradiction.

Case 7. $\bar{G}$ is isomorphic to $\mathrm{PSL}_{2}\left(r^{h}\right)$, where $r^{h} \geq 4$. If $r$ is even, then, by the same discussion as we had in Case 6, we produce a contradiction. So we may assume $r$ is odd. If $r \nmid q$, then $r^{h} \mid(q \pm 1)$ and $q \mid\left(r^{h} \pm 1\right)$. Thus $r^{h}-1 \leq q \leq r^{h}+1$ and so $q=r^{h} \pm 1$, a contradiction. Therefore $r^{h}=q$ and hence $\bar{G} \cong \mathrm{PSL}_{2}(q)$.

Lemma 2.18. Let $\pi(q-\nu)=\{2\}$. Then $\bar{G} \cong \operatorname{PSL}_{2}(q)$.

Proof. Let $q-\nu=2^{\alpha}$, for some integer $\alpha$. Then either $q=p$ is prime, or $q=9$. First, assume $q=p$ is prime. We show that $\bar{G}$ is a Zassenhaus group of degree $p+1$. We know that $|\bar{G}|=p\left(p^{2}-1\right) / 2$.

Now we claim that either $n_{p}(\bar{G})=p+1$ or $n_{p}(\bar{G})=1$. Let $n_{p}(\bar{G})=m t=k p+1$, where $m, t$ and $k$ are integers such that $m \mid(p+1)$ and $t \mid(p-1) / 2$. If either $m=p+1$ or $m=1$, then $t=1$, since $t \equiv 1(\bmod p)$. So we may assume $1<m \leq(p+1) / 2$. Then we can write $t=(p-1) / 2 m^{\prime}$, where $t \geq 2$ and hence $2 m^{\prime} \leq(p-1) / 2$, since otherwise $1<n_{p}(\bar{G})<p+1$ and this is impossible. Considering $m(p-1) / 2 m^{\prime}=k p+1$, we have $p \mid 2 m^{\prime}+m$. Using the fact $2 m^{\prime} \leq(p-1) / 2$ and $m \leq(p+1) / 2$, we conclude that $2 m^{\prime}=(p-1) / 2$ and $m=(p+1) / 2$. Thus $k p+1=p+1$ as we claimed.

If $n_{p}(\bar{G})=1$, then $\bar{G}=H \ltimes P$, where $P \in \operatorname{Syl}_{p}(\bar{G})$ and $H$ is a $p$-complement of $\bar{G}$. We know that $C_{H}(x)=1$ for every $x \in P$, by Lemma 2.15 (ii). Hence $\bar{G}$ is a Frobenius group, which contradicts $\left(p^{2}-1\right) / 2=|H| \mid p-1$. So $n_{p}(\bar{G})=p+1$.

Now, let $\Omega=\operatorname{Syl}_{p}(\bar{G})$. Obviously $\bar{G}$ acts on $\Omega$ transitively and the stabilizer $\bar{G}_{P}=N_{\bar{G}}(P)$, for every $P \in \Omega$. Hence $N_{\bar{G}}(P)=H \ltimes P$, where $H$ is a subgroup of $\bar{G}$ whose order is $(p-1) / 2$. Since $P \cap N_{\bar{G}}\left(P_{0}\right)=P \cap P_{0}=1$, for every $P_{0} \in \Omega-\{P\}$, we infer that $P$ acts on $\Omega-\{P\}$ transitively. So the stabilizer of $P, \bar{G}_{P}$, acts on $\Omega-\{P\}$ transitively, which implies that $\bar{G}$ is doubly transitive. It means $\left|\bar{G}_{P}: \bar{G}_{P_{0}} \cap \bar{G}_{P}\right|=p$ for every $P \neq P_{0} \in \Omega$.

So we can say that for every $P \neq P_{0} \in \Omega, \bar{G}_{P} \cap \bar{G}_{P_{0}}$ is a $p$-complement of $N_{\bar{G}}(P)$ and so we may assume $\bar{G}_{P} \cap \bar{G}_{P_{0}}=H$, for some $p$-complement $H$ of $N_{\bar{G}}(P)$. Note that $N_{\bar{G}}(P)$ is a Frobenius group, by Lemma 2.15 (ii), and so $N_{\bar{G}}(P)=\left(\bigcup_{g \in P} H^{g}\right) \bigcup P$. Hence every $p^{\prime}$-element in $N_{\bar{G}}(P)$ fixes at least one Sylow $p$-subgroup in $\Omega-\{P\}$. Thus, if we consider the action of $\bar{G}_{P}$ on $\Omega-\{P\}$, then we have $|\operatorname{Fix}(h)| \geq 1$, for every $p^{\prime}$-element of $N_{\bar{G}}(P)$. As we discussed, non-identity elements in $P$ does not fix any element in $\Omega-\{P\}$. Now considering the following equation,

$$
\left|N_{\bar{G}}(P)\right|=p+\sum_{h \in N_{\bar{G}}(P)-P}|\operatorname{Fix}(h)|
$$

we will have $|\operatorname{Fix}(h)|=1$. Therefore the elements in $\bar{G}$ fixes at most 2 points in $\Omega$.

Let $K \unlhd \bar{G}$ such that $K$ acts on $\Omega$ transitively and also $K_{P}=1$, for every $P \in \Omega$. Since the action of $K$ on $\Omega$ is transitive, then $\left|K: K_{P}\right|=|\Omega|=p+1$, for every $P \in \Omega$. Therefore $|K|=p+1$. Now consider $P \ltimes K$. By Lemma 2.15 (ii), $C_{P}(k)=1$, for every $k \in K$. Hence $P \ltimes K$ is a Frobenius group. This implies that $K$ is nilpotent and so $K$ is a subgroup of either a Hall $\pi(q-\nu)$-subgroup, or a Hall $\pi((q+\nu) / 2)$-subgroup. So we conclude that $|K|=p+1=q-\nu=2^{\alpha}$. Now consider $\bar{G}=H \ltimes K$, where $H$ is a 2-complement of $\bar{G}$. Again we can see that $\bar{G}$ is a Frobenius group, which is contradicting $|H| \nmid|K|-1=p$. Hence $\bar{G}$ does not have any regular normal subgroup. Therefore our claim is proved and $\bar{G}$ is a Zassenhaus group of degree $p+1$. Now using Theorem 2.1 and from Lemma 2.15 (i), we have $\bar{G}=\mathrm{PSL}_{2}(p)$, as wanted.

Secondly, let $q=9$ and so $|\bar{G}|=9.5 .8$. Thus either $n_{3}(\bar{G})=1, n_{3}(\bar{G})=4, n_{3}(\bar{G})=40$ or $n_{3}(\bar{G})=10$. Similar to the first case we conclude that $n_{3}(\bar{G}) \neq 1$. Let $n_{3}(\bar{G})=4$. We know that $\bar{G}$ acts transitively on $\operatorname{Syl}_{3}(\bar{G})$. So for $P \in \operatorname{Syl}_{3}(\bar{G}), \bar{G}_{P}=N_{\bar{G}}(P)=H \ltimes P$, where $|H|=10$. Moreover $N_{\bar{G}}(P)$ is a Frobenius group, which contradicts $10 \nmid 9-1$. If $n_{3}(\bar{G})=40$, then $C_{\bar{G}}(P)=N_{\bar{G}}(P)$, for $P \in \operatorname{Syl}_{3}(\bar{G})$. 
Therefore $\bar{G}$ is 3-nilpotent and so $\bar{G}=P \ltimes K$, where $K$ is the 3 -complement of $\bar{G}$. As we discussed before $\bar{G}$ is a Frobenius group and hence $K$ is nilpotent, a contradiction. Hence $n_{3}(\bar{G})=10$.

We claim that every two Sylow 3-subgroups have a trivial intersection. On the contrary, assume there exist $P, R \in \operatorname{Syl}_{3}(\bar{G})$, such that $S=(P \cap R)$ has index 3 in $P$. By Lemma 2.4, we have $\left|N_{\bar{G}}(S)\right|=9 t$, where $t \mid 40$ and $t \geq 4$. Considering Lemma 2.15 (ii), we have $\left|C_{\bar{G}}(S)\right|=9$. By using Normalizer-Centralizer Theorem we have $t \leq 2$, a contradiction. Therefore our claim is proved.

Now using the fact that every two Sylow 3 -subgroups have a trivial intersection, similar to the first case, we obtain $G$ is a Zassenhaus group of degree 10 and so we have $G \cong \operatorname{PSL}_{2}(9)$, which is our desired result.

The proof of Theorem 2.10 is an immediate consequence of Lemmas 2.17 and 2.18.

Theorem 2.19. Let $G$ be a finite group. If $\operatorname{cs}(G)=\operatorname{cs}\left(\mathrm{PSL}_{2}(q)\right)$, with $q \geq 7$ odd, then $G \cong \mathrm{PSL}_{2}(q) \times A$, where $A$ is an abelian group.

Proof. By Theorem 2.10, it is enough to prove that either $\mathbf{Z}(G)=1$ or $\mathbf{Z}(G)$ is a direct factor of $G$. We argue by minimal counterexample. So we assume $G$ is a group with minimal order such that $\operatorname{cs}(G)=\operatorname{cs}\left(\mathrm{PSL}_{2}(q)\right)$ and $\mathbf{Z}(G)$ is not trivial and it is not a direct factor of $G$.

By Theorem 2.10 we have $G^{\prime} \mathbf{Z}(G)=G$ and $G^{\prime} /\left(\mathbf{Z}(G) \cap G^{\prime}\right) \cong \operatorname{PSL}_{2}(q)$. Note that $G^{\prime} \cap \mathbf{Z}(G)=\mathbf{Z}\left(G^{\prime}\right)$, since otherwise if $x \in \mathbf{Z}\left(G^{\prime}\right)-\mathbf{Z}(G)$, then $x\left(G^{\prime} \cap \mathbf{Z}(G)\right) \in \mathbf{Z}\left(G^{\prime} /\left(G^{\prime} \cap \mathbf{Z}(G)\right)\right)$, a contradiction. Now by Lemma 2.5, we conclude that $\operatorname{cs}(G)=\operatorname{cs}\left(G^{\prime}\right)$.

We claim that $G=G^{\prime}$. Assume, on the contrary $G^{\prime}<G$. But since $G$ is a minimal counterexample such that its center is not trivial and is not a direct factor of $G$ and $\operatorname{cs}(G)=\operatorname{cs}\left(\operatorname{PSL}_{2}(q)\right)$, either $\mathbf{Z}\left(G^{\prime}\right)=\mathbf{Z}(G) \cap G^{\prime}=1$ or $G^{\prime}=T \times \mathbf{Z}\left(G^{\prime}\right)$ and $T \cong \mathrm{PSL}_{2}(q)$.

Assume the former case occurs. Then $G \cong G^{\prime} \times \mathbf{Z}(G) \cong \mathrm{PSL}_{2}(q) \times \mathbf{Z}(G)$, which is not possible by our assumption on $G$. So we may assume the latter case holds. Therefore $G=G^{\prime} \mathbf{Z}(G) \cong\left(T \times \mathbf{Z}\left(G^{\prime}\right)\right) \mathbf{Z}(G) \cong$ $T \times \mathbf{Z}(G)$ which again contradicts our assumption on $G$. Hence $G^{\prime}=G$.

Now since $G$ is perfect and $G / \mathbf{Z}(G)$ is simple, $G$ is a quasi-simple group and so $\mathbf{Z}(G) \leq M\left(\operatorname{PSL}_{2}(q)\right)$, where $M\left(\mathrm{PSL}_{2}(q)\right)$ is the Schur multiplier of finite simple group $\mathrm{PSL}_{2}(q)$. If $q \neq 9$, then $\left|M\left(\operatorname{PSL}_{2}(q)\right)\right|=$ 2 and since $\mathbf{Z}(G)$ is not trivial, we have $\mathbf{Z}(G)=M\left(\mathrm{PSL}_{2}(q)\right)$. Hence $G$ is isomorphic to the unique Schur cover of $\mathrm{PSL}_{2}(q)$ which is $G \cong \mathrm{SL}(2, q)$. By considering the set of conjugacy class sizes of $\mathrm{SL}(2, q)$, we get a contradiction.

Now if $q=9$, then $G$ is a quotient of the Schur representation of $\mathrm{PSL}_{2}(9)$. In fact, if $6 \cdot A_{6}$ denotes the Schur representation of $\mathrm{PSL}_{2}(9)$, then $G \cong 6 \cdot A_{6}, 3 \cdot A_{6}$ or $\mathrm{SL}_{2}(9)$. By checking the conjugacy class sizes of these groups, we produce a contradiction.

\section{Acknowledgements}

The authors are grateful to the referees for their comments and suggestions.

\section{References}

[1] N. Ahanjideh, On Thompson's Conjecture for finite simple groups, J. Algebra 344 (2011), 205-228.

[2] E. Alemany, A. Beltrán, M. J. Felipe, Finite groups with two p-regular conjugacy class lengths, II, Bull. Austral. Math. Soc. 79 (2009), 419-425.

[3] R. Baer, Einfache Partitionen endlicher Gruppen mit nicht-trivialer Fittingscher Untergruppe, (German) Arch. Math. (Basel) 12 (1961), 81-89.

[4] R. Baer, Partitionen endlicher Gruppen, (German) Math. Z. 75 (1961), 333-372. 
[5] A. Beltrán, M.J. Felipe, Finite groups with four conjugacy class sizes, Comm. Algebra 39 (2011), $1260-1272$.

[6] A. R. Camina. Arithmetical conditions on the conjugacy class numbers of a finite group, J. London Math. Soc. 2 (1972), 127-132.

[7] J.H. Conway, R.T. Curtis, S.P. Norton, R.A. Parker, R.A. Wilson, Atlas of finite groups, Oxford University Press, Oxford 1985.

[8] S. Dolfi, M. Herzog, E. Jabara, Finite groups whose noncentral commuting elements have centralizers of equal size, Bull. Aust. Math. Soc. 82 (2010), 293-304.

[9] B. Huppert, N. Blackburn, Finite Groups III, Springer-Verlag Berlin Heidelberg, 1982.

[10] D.J.S. Robinson, A course in the group theory, Springer-Verlag New York Heidelberg Berlin, 1982.

[11] J. Rebmann, F-Gruppen, Arch. Math. 22 (1971), 225-230.

[12] M. Suzuki, On a finite group with a partition, Arch. Math. (Basel) 12 (1961), 241-254. 